\title{
Design and Characterization of a Hypervelocity Expansion Tube Facility
}

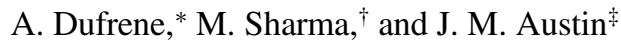 \\ University of Illinois at Urbana-Champaign, Urbana, Illinois 61801
}

DOI: $\underline{10.2514 / 1.30349}$

\begin{abstract}
We report on the design and characterization of a 152-mm-diam expansion tube capable of accessing a range of high-enthalpy test conditions with Mach numbers up to 7.1. Expansion tubes have the potential to offer a wide range of test flow conditions as gas acceleration is achieved through interaction with an unsteady expansion wave rather than expansion through a fixed-area-ratio nozzle. However, the range of test flow conditions is limited in practice by a number of considerations such as a short test time and large-amplitude flow disturbances. We present a generalized design strategy for small-scale expansion tubes. As a starting point, ideal gasdynamic calculations for optimal facility design to maximize test time at a given Mach number test condition are presented, together with a correction for the expansion-head reflection through a nonsimple region. A compilation of practical limitations that have been identified for expansion tube facilities, such as diaphragm rupture and flow-disturbance minimization, is then used to map out a functional design parameter space. Experimentally, a range of test conditions are verified through pitot pressure measurements and analysis of schlieren images of flow over simple geometries. To date, there has been good agreement between theoretical and experimental results.
\end{abstract}

\section{Introduction}

G ROUND test facilities that can achieve hypersonic flight conditions are critical to the further development of hypersonic airbreathing propulsion systems, reentry vehicles, and planetary probes for space exploration. The simulation of hypersonic flows in which chemical and thermal real-gas effects become important (commonly referred to as hypervelocity flows; see, for example, Hornung [1]) requires high-stagnation-enthalpy conditions that can be achieved in arc- or combustion-heated facilities or in impulse facilities [2,3]. Existing hypervelocity impulse facilities include large-scale reflected shock tunnels such as T5 at the California Institute of Technology [4], the Large Energy National Shock Tunnel facilities at CUBR $\bar{C}$ [5], the High-Enthalpy Wind Tunnel, Goettingen at the DLR, German Aerospace Center [6], and the HighEnthalpy Shock Tunnel at the National Aerospace Laboratories in Kakuda [7] and expansion tube facilities such as NASA's HYPULSE facility [8], JX-1 at Tohoku University [9], and the $\mathrm{X}$-series facilities at the University of Queensland [10].

Recently, some smaller-scale facilities have been developed. Smaller-scale facilities avoid the longer test-preparation times needed in some of the larger-scale tunnels mentioned earlier. A 140$\mathrm{mm}$-diam expansion tube facility covering vehicle flight Mach numbers of 4 to 9 for scramjet combustion studies was recently built at Stanford University [11]. In the present paper, we report on the design and characterization of a new 152-mm-diam small-scale expansion tube facility that may be used to achieve a range of test-gas Mach numbers from 3.0 to 7.1.

An expansion tube has several advantages and disadvantages in comparison with the more common reflected shock tunnel [1-3]. In an expansion tube, the initial shock interacts with a contact surface,

Presented as Paper 1327 at the 45th AIAA Aerospace Sciences Meeting and Exhibit, Reno, NV, 8-11 January 2007; received 8 February 2007; revision received 14 June 2007; accepted for publication 16 June 2007. Copyright (c) 2007 by the University of Illinois at Urbana-Champaign. Published by the American Institute of Aeronautics and Astronautics, Inc., with permission. Copies of this paper may be made for personal or internal use, on condition that the copier pay the $\$ 10.00$ per-copy fee to the Copyright Clearance Center, Inc., 222 Rosewood Drive, Danvers, MA 01923; include the code 0748-4658/07 \$10.00 in correspondence with the CCC.

*Former Graduate Student, Department of Aerospace Engineering. Member AIAA.

${ }^{\dagger}$ Graduate Student, Department of Aerospace Engineering.

\#Assistant Professor, Department of Aerospace Engineering. Senior Member AIAA. generating an unsteady expansion that further accelerates the test gas (see Sec. II). Theoretically, a wide range of test conditions with decreased freestream dissociation can be achieved by varying initial pressures $[12,13]$. In contrast, in a shock tunnel, chemical and vibrational freezing commonly occur in the nozzle, and the Mach number of the test gas can only be varied by exchanging the nozzles. However, expansion tubes tend to have shorter test times and increased boundary-layer effects, because the test condition is some distance downstream of the initial shock. In practice, expansion tube operating ranges have been limited by secondary-diaphragm considerations and the appearance of large-amplitude test flow disturbances.

We present a design procedure for small-scale expansion tubes. Ideal test condition calculations based on unsteady gasdynamics are first presented, with particular focus on the important problem of short test times. We experimentally investigate optimizing the secondary-diaphragm material, thickness, and burst mechanism for the initial pressures used in an expansion tube facility. We apply Paull and Stalker's [17] criteria for disturbance minimization, together with other practical design considerations, to identify a useful parameter space for the operation of a small-scale expansion tube facility. A range of test conditions up to Mach 7.1 with suitable core flow size have been experimentally verified in this facility through pitot pressure measurements and the analysis of schlieren images of flow over simple geometries. Experimental and theoretical calculations are compared for a range of test conditions.

\section{Gasdynamic Processes in the Expansion Tube}

The flow processes in an expansion tube are complex. Nevertheless, simple one-dimensional, inviscid, unsteady wave interactions may be used to calculate the ideal test conditions, including thermodynamic state, flow velocity, and test time as a starting point for facility design.

\section{A. Calculation of the Thermodynamic State}

The expansion tube consists of three sections (a driver, driven, and expansion section) separated by two diaphragms. A sample $x-t$ diagram for a Mach 5.1 test flow is shown in Fig. 1. The driver and driven sections operate as a standard shock tube. The strength of the primary left-facing expansion wave is dependent on the initial pressures, but is generally sufficiently strong to accelerate the gas past sonic velocities. The head of the primary expansion wave will 


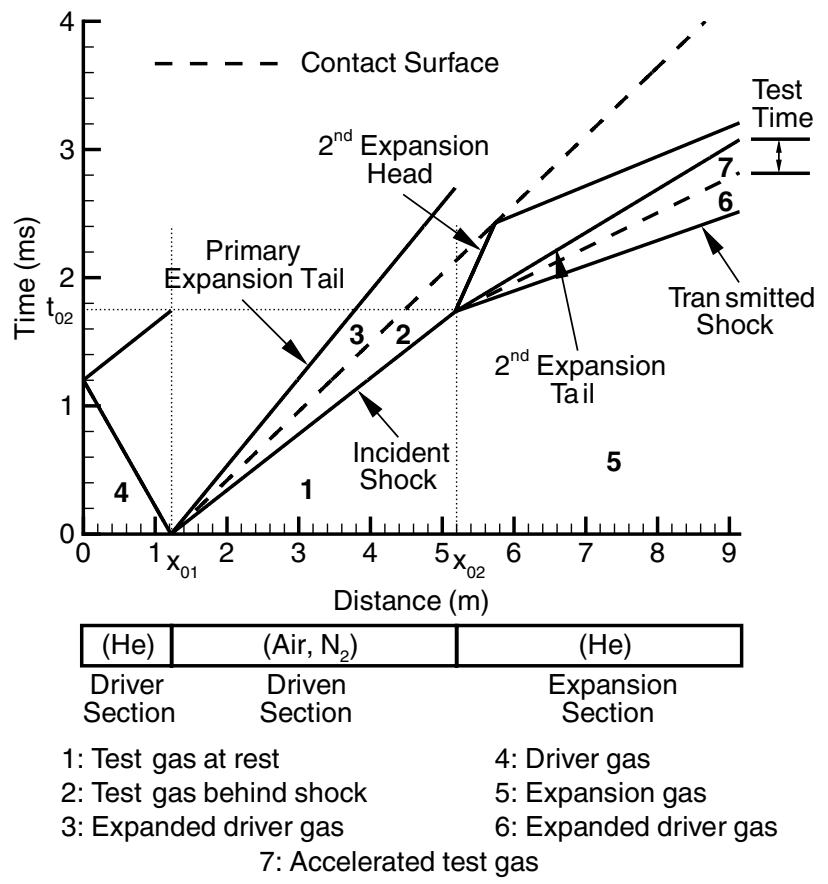

Fig. 1 Sample $x-t$ diagram of the calculated gasdynamic processes for a Mach 5.1 test flow in the HET facility $\left(p_{4}=6250 \mathrm{kPa}, p_{1}=1.1 \mathrm{kPa}\right.$, and $p_{5}=0.1 \mathrm{kPa}$ ); driver and expansion sections are filled with helium and the driven section with air; calculations assume perfect gas.

reflect from the upstream end of the tube and the tail will trail the primary contact surface.

The secondary diaphragm, assumed in this initial theoretical analysis to be thin and break instantaneously upon arrival of the incident shock, is represented as a contact surface. Pressure-velocity $p-u$ polars are used to calculate states 6 and 7 (see Fig. 1) resulting from incident-shock/contact surface interaction. A shock is transmitted and the expansion wave between regions 2 and 7 can be either right-facing or left-facing. From the $p-u$ plane in Fig. 2 , it is apparent that the right-facing wave has no solution, and so the wave is a left-facing expansion. The head and tail of the expansion have positive slopes, because the flow in state 2 is supersonic and to the right. State 7 is the test gas that was further accelerated beyond the postshock state 2 by the unsteady secondary expansion.

The secondary expansion wave will interact with the contact surface between states 2 and 3 , resulting in reflected and transmitted waves. Examining the $p-u$ diagram shows that the reflected wave is also an expansion. Thus, a nonsimple region is created in which the two expansion waves interact.

\section{B. Test-Time Calculation}

Test time varies with the initial conditions of the expansion tube and can be estimated from an $x-t$ diagram. The test time begins with the arrival of the state 6-7 contact surface, and it is terminated by the arrival of either 1) the head of the reflected secondary expansion wave, 2) the tail of the secondary expansion wave, or 3) the arrival of the primary expansion from the driver, whichever occurs first. We consider cases 1 and 2 first. For these two cases, the maximum test time corresponds to the case in which the reflected head and the tail of the secondary expansion fan arrive at the end of the tube at the same time.

The interaction of the incident and reflected secondary expansion waves creates a nonsimple region. We may use the method of characteristics to solve for the trajectory of the leading characteristic of the reflected expansion as it passes through the incident expansion. A more detailed description is given by Wintenberger et al. [14]. Assuming an isentropic expansion, an ordinary differential equation for the passage of the first reflected characteristic through a leftfacing expansion can be derived in terms of a similarity variable $\eta=x(t) / c_{2} t$ :

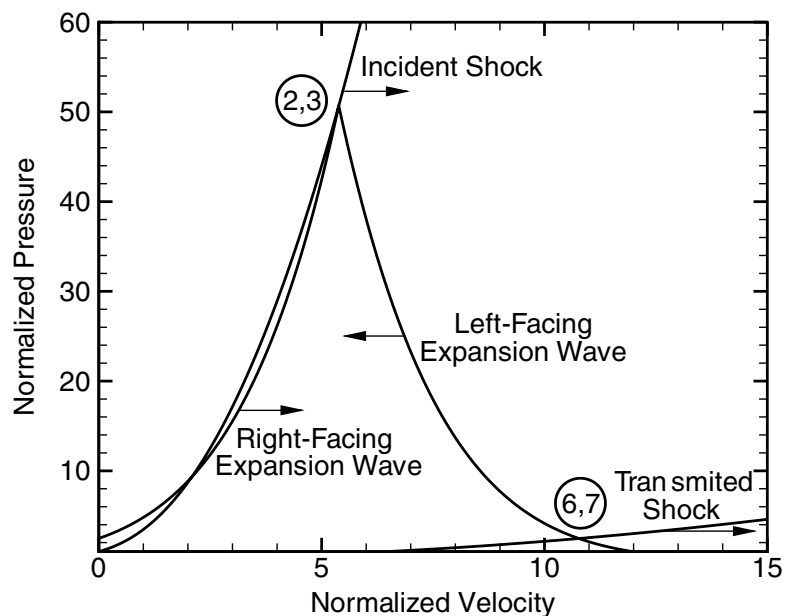

Fig. 2 Normalized pressure $p / p_{1}$ and velocity $u / c_{1}$ polar calculation for incident-shock interaction, with the secondary diaphragm represented as a contact surface; $p_{1}$ and $c_{1}$ are the pressure and sound speed of the gas at state 1, respectively; calculations are performed for the same conditions as in Fig. 1.

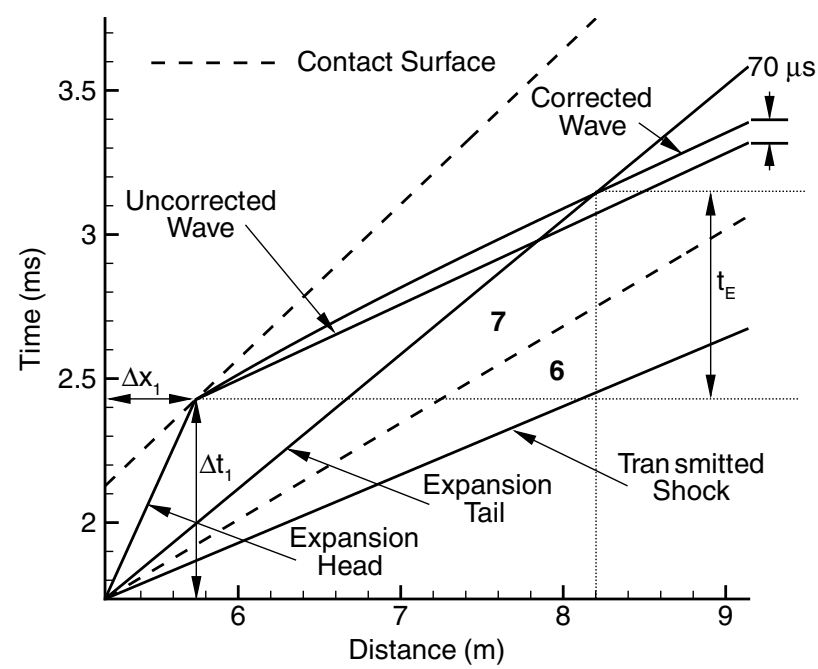

Fig. 3 Detail from the $x-t$ diagram from the secondary diaphragm, showing the effect of the reflected wave correction for the interaction with the incident expansion wave [14]; origin of the $x$ axis is the arrival of the incident shock at the secondary-diaphragm station, $x_{02}=5.18 \mathrm{~m}$ (see Fig. 1); calculations are performed for the following conditions: $p_{4}=6250 \mathrm{kPa}, p_{1}=1.1 \mathrm{kPa}$, and $p_{5}=0.5 \mathrm{kPa}$.

$$
t \frac{d \eta}{d t}+\frac{2\left(\gamma_{2}-1\right)}{\gamma_{2}+1}\left(\eta-\frac{u_{2}}{c_{2}}-\frac{2}{\gamma_{2}-1}\right)=0
$$

which has the solution

$$
\eta(t)=\frac{u_{2}}{c_{2}}+\frac{2}{\gamma_{2}-1}\left[1-\frac{\gamma_{2}+1}{2}\left(\frac{t}{\Delta t_{1}}\right)^{\frac{2\left(1-\gamma_{2}\right)}{\gamma_{2}+1}}\right]
$$

where $\Delta t_{1}$ is the time between the arrival of the initial shock at the secondary-diaphragm location $x_{02}$ and the intersection between the first contact surface and the reflected expansion head, as indicated in Fig. 3 (the origin of Fig. 3 is $t_{02}$ and $x_{02}$ in Fig. 1), $u_{2}$ is the gas velocity, $c_{2}$ is the gas sound speed, and $\gamma_{2}$ is the specific gas ratio, which is assumed to be constant. Subscripts refer to the state, as shown in Fig. 1.

The similarity solution must be referenced from the arrival of the initial shock at the secondary-diaphragm location $x_{02}$. Figure 3 also shows the location of the leading characteristic of the reflected expansion with and without the correction for the interaction with the 
Table 1 Sample theoretical test conditions 1 through 4 for expansion tube operation

\begin{tabular}{lcccc}
\hline \hline Condition & 1 & 2 & 3 & 4 \\
\hline Mach number & 7.27 & 5.21 & 4.00 & 3.60 \\
Static temperature, $\mathrm{K}$ & 588 & 1228 & 1392 & 930 \\
Static pressure, $\mathrm{kPa}$ & 2.92 & 3.10 & 9.67 & 12.67 \\
Velocity, $\mathrm{m} / \mathrm{s}$ & 3540 & 3660 & 3000 & 2210 \\
Density, $\mathrm{kg} / \mathrm{m}^{3}$ & 0.017 & 0.008 & 0.024 & 0.047 \\
Test time, $\mu \mathrm{s}$ & - & - & 335 & 447 \\
Corrected test time-a,$\mu$ s & 180 & 267 & 419 & 576 \\
Initial pressures, $\mathrm{kPa}$ & & & & \\
Driver section & 6250 & 6250 & 5000 & 1250 \\
Driven section & 6.0 & 1.1 & 1.65 & 3.0 \\
Expansion section & 0.1 & 0.1 & 0.45 & 1.0 \\
\hline \hline
\end{tabular}

The correction to the test time due to expansion wave interaction [see Eq. (2)] only applies when the test time is terminated by the arrival of the reflected expansion head (conditions 3 and 4 ).

incident expansion. It was found that there was an error of up to $35 \%$ in calculating the test time for the reflected-head-limiting case when this correction is neglected. Table 1 presents some sample theoretical run conditions in which this correction is important. The time $t_{E}$, which corresponds to the intersection between the secondary expansion tail and the reflected secondary expansion head in the $x-t$ plane, can then be found from Eq. (2) as

$$
t_{E}=\Delta t_{1}\left[\left(\frac{c_{7}}{c_{2}}\right)^{\frac{\gamma_{2}+1}{2\left(1-\gamma_{2}\right)}}-1\right]
$$

Figure 3 illustrates the situation in which the test time is limited by the reflected expansion head, whereas Fig. 1 is an example of an expansion-tail-limited solution. The maximum test time will occur when both of these waves arrive simultaneously at the end of the expansion section. Using the notation of Fig. 3 , the maximum test time $t_{\text {test,max }}$ would then be given as

$$
t_{\text {test, } \max }=\Delta t_{1}+t_{E}-\Delta t_{2}
$$

where $\Delta t_{2}$ is defined as the time difference between the second contact surface arrival at the optimal-tube-length end and the initial shock arrival at the secondary diaphragm. The optimal length of the facility expansion section for maximum test time $L_{\mathrm{EXP}}$ can then be selected:

$$
L_{\mathrm{EXP}}=\left(u_{7}-c_{7}\right)\left(t_{E}+\Delta t_{1}\right)
$$

The optimal expansion-section length as a function of the expansionto-driven initial pressure ratio $p_{5} / p_{1}$ is shown in Fig. 4 for different $p_{4} / p_{1}$ values. Lower $p_{5} / p_{1}$ values result in higher test flow Mach numbers and require longer expansion sections for maximum test time. It can be seen that the optimal expansion-length parameter is insensitive to the driver-to-driven initial pressure ratio $p_{4} / p_{1}$. The horizontal line in Fig. $\underline{4}$ denotes the expansion-section length selected for the current hypervelocity expansion tube (HET) design. Solutions that fall below this line correspond to the test time being limited by the reflected secondary expansion head and those that fall above it are restricted by the secondary expansion tail.

\section{Design and Test-Time Limitations}

Significant test flow disturbances were found to occur in the first expansion tubes $[15,16]$. It was observed that the test flow was rendered completely unacceptable, due to the presence of highfrequency fluctuations. Paull and Stalker [17] theoretically showed that the dominant part of this noise can be modeled as acoustic lateral waves from the driver gas, which are focused into a narrowfrequency band on transmission through the expansion. They also showed that these waves can be strongly minimized by decreasing the primary-contact-surface sound speed ratio $c_{3} / c_{2}$. An analytical relationship for this sound speed ratio is

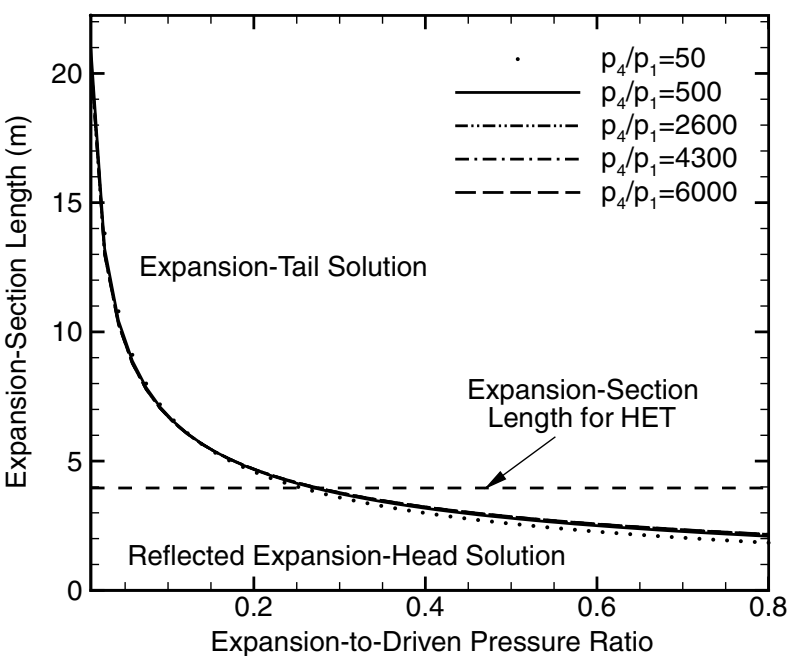

Fig. 4 Optimal expansion-section length $L_{\mathrm{EXP}}$ for maximum test time as a function of expansion-to-driven-section initial pressure ratio $p_{5} / p_{1}$ for different $p_{4} / p_{1}$; air is the test gas in the driven section; driver and expansion sections are filled with helium.

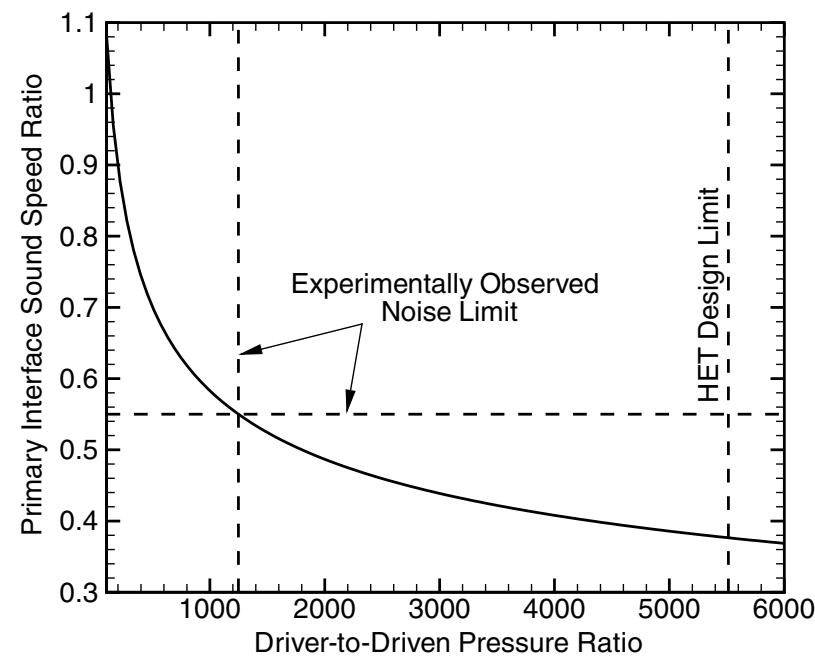

Fig. 5 Primary interface sound speed ratio $c_{3} / c_{2}$ as a function of driver-to-driven initial pressure ratio $p_{4} / p_{1}$; air is the test gas in the driven section; driver and expansion sections are filled with helium.

$$
\frac{c_{3}}{c_{2}}=\frac{\left(p_{4} / p_{1}\right)^{\left(1-2 \gamma_{4}\right) / 2 \gamma_{4}}\left(c_{4} / c_{1}\right)}{\sqrt{\frac{\left(2 / M_{s}^{2}\right)+\gamma_{1}-1}{\gamma_{1}+1}}\left[1+\frac{2 \gamma_{1}\left(M_{s}^{2}-1\right)}{\gamma_{1}+1}\right]^{1 / 2 \gamma_{4}}}
$$

where the shock Mach number $M_{s}$ comes from the implicit shock tube relationship. For $\gamma_{4}=1.667$ and $\gamma_{1}=1.397$, Fig. 5 shows the relationship between the primary-contact-surface sound speed ratio and $p_{4} / p_{1}$. The result shows that the sound speed ratio, and therefore the test flow disturbance, can be reduced by increasing the driver-todriven-gas pressure ratio. Paull and Stalker do not suggest a value for the sound speed ratio that produces acceptable amounts of noise, instead identifying high-enthalpy and low-enthalpy conditions as $c_{3} / c_{2}$ as less than or greater than one, respectively. Low-enthalpy conditions were found to be unacceptably noisy. Based on test-time pitot data obtained in the current HET facility, it is suggested that the upper limit for $c_{3} / c_{2}$ be set at 0.55 . The $p_{7 \text {,pitot }}$ value is obtained from the pressure trace by averaging over the constant-pressure region that follows the arrival of the contact surface (see Sec. III.B). The noise limit of 0.55 corresponds to the point at which the fluctuations are at most $\pm 50 \%$ that of the mean value within the test-time section of the pitot trace. This value will in turn correspond to a minimum allowable $p_{4} / p_{1}$ value. This value is $p_{4} / p_{1}=1248$ for the current 


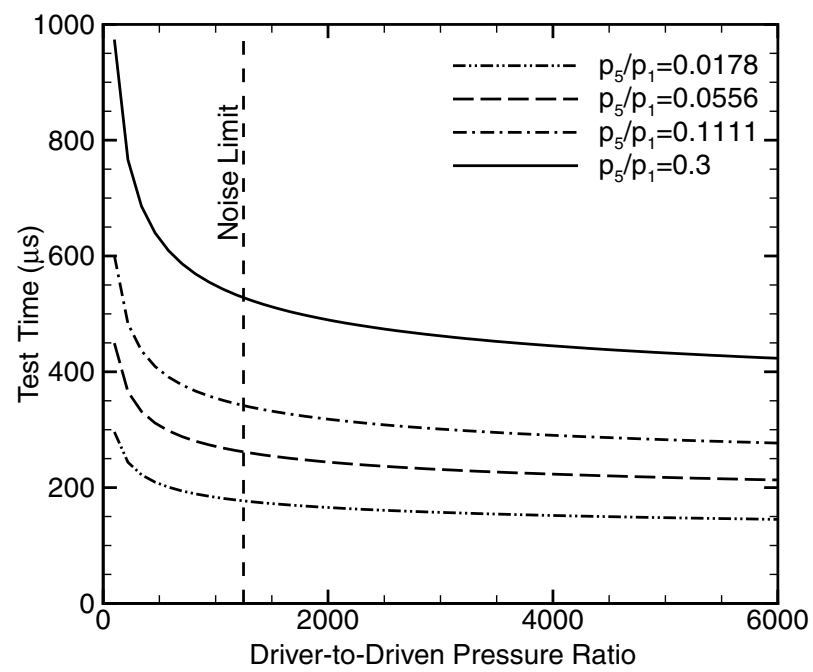

Fig. 6 Test time as a function of driver-to-driven initial pressure ratio $p_{4} / p_{1}$ for different $p_{5} / p_{1}$ values; air is the test gas in the driven section; driver and expansion sections are filled with helium.

facility. Figure 5 shows the regime in which the HET facility can operate and is bounded by the experimentally observed acceptable sound speed ratio. The upper bound on the pressure ratio is set by the design yield strength of the driver tube section as $5.5 \mathrm{MPa}$, assuming a driven pressure of $1.0 \mathrm{kPa}$.

The dependence of the test time $t_{\text {test }}$ on $p_{5} / p_{1}$ for different $p_{4} / p_{1}$ values is illustrated in Fig. 6 . For a given $p_{5} / p_{1}$ value, test time can be increased by decreasing the $p_{4} / p_{1}$ value. However, there is a limitation placed upon the minimum $p_{4} / p_{1}$ value, due to the flowdisturbance considerations of the $c_{3} / c_{2}$ ratio, as discussed earlier. The limit for a sound speed ratio of 0.55 is shown as the vertical line in the figure. Acceptable test conditions are to the right of this line. One can see that within this acceptable regime, the dominant influencing factor is that of the $p_{5} / p_{1}$ value rather than the $p_{4} / p_{1}$ value. Thus, the noise limitation tends to dictate the choice of $p_{4} / p_{1}$, rather than test-time considerations. For a certain run condition, this vertical line also indicates the maximum allowable test time. A great advantage of expansion tubes is the range of test flow Mach numbers that can be accessed by simply varying the initial pressures. The HET facility was designed to produce a range of test flows with Mach numbers from 3.0 to 7.1, as shown in Fig. 7. We have selected three run conditions for aerodynamic studies (air-1, air-2, and air-3), as summarized in Table 2. The flow Mach number $M_{7}$ can essentially be selected using the expansion-to-driven pressure ratio $p_{5} / p_{1}$. Figure 7 shows that the Mach number is increasing with decreasing $p_{5} / p_{1}$ for all selected values of the driver-to-driven pressure ratio $p_{4} / p_{1}$. For higher $p_{4} / p_{1}$ ratios, the Mach number is very insensitive to this ratio. Conditions with lower $p_{4} / p_{1}$ ratios exhibit more sensitivity; however, these low pressure ratios may be eliminated as potential run conditions due to noise minimization considerations. It is evident that the dominant factor for both the expansion tube sizing and testsection Mach number is the $p_{5} / p_{1}$ ratio. Figure 7 also shows that to maintain high Mach number operation for the current facility, $p_{5} / p_{1}$ should be kept below 0.3 . As previously mentioned, in the results from Fig. 6 , one can see that to increase the test time for a given $p_{4} / p_{1}$ value, the $p_{5} / p_{1}$ ratio must be increased. Figure 7 , however, shows that an increase in $p_{5} / p_{1}$ causes a reduction in $M_{7}$. This demonstrates the need for a working balance within the design process that takes into account the restrictions, dominant factors, and fundamental goals of the facility.

So far in this discussion, we have only considered the arrival of the secondary expansion tail or the reflected expansion head as the limiting conditions for the test time. As discussed earlier, a third possibility is the arrival of the primary expansion wave. As seen in Fig. 1, this possibility includes the arrival of the expansion reflected from the driver wall and its tail. The absolute times that the reflected primary driver expansion head, primary driver expansion tail, and the second contact surface arrive at the end of the tube are denoted as $t_{r d h}$,

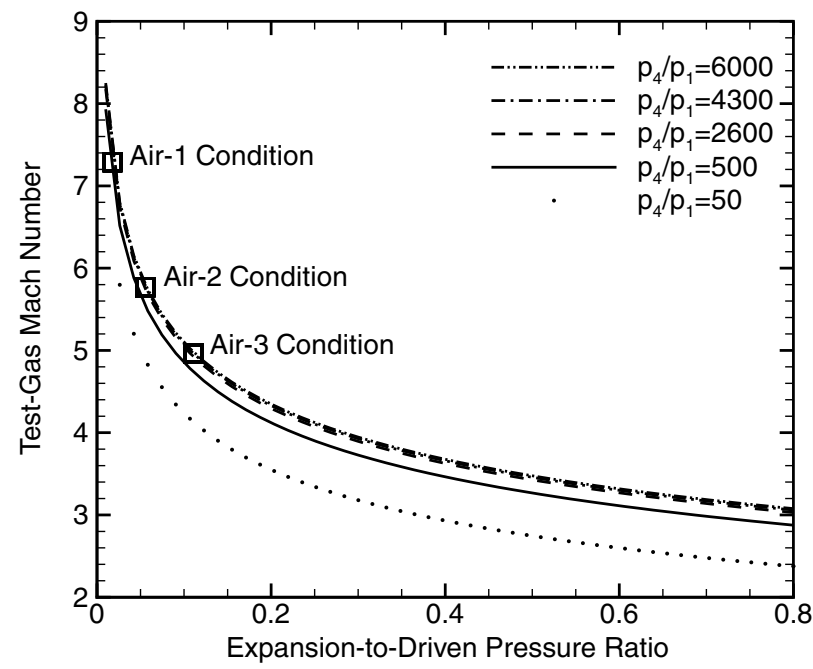

Fig. 7 Test-gas Mach number $M_{7}$ as a function of expansion-to-drivensection initial pressure ratio $p_{5} / p_{1}$ for different $p_{4} / p_{1}$ values; air is the test gas in the driven section; driver and expansion sections are filled with helium.

$t_{d t}$, and $t_{2}$, respectively. A ratio between $t_{r d h}$ and $t_{2}$ and the test time $t_{\text {test }}$ for a certain run condition is given as

$$
\beta=\frac{t_{\text {test }}}{t_{r d h}-t_{2}}
$$

A ratio between $t_{d t}$ and $t_{2}$ and the test time is defined as

$$
\alpha=\frac{t_{\text {test }}}{t_{d t}-t_{2}}
$$

For the reflected primary driver expansion condition to be considered, the time between the arrival of the second contact surface and the reflected primary driver expansion head should be of the same order as the test time $t_{\text {test }}$, such that $\beta \sim 1$. The variation of $\beta$ with $p_{4} / p_{1}$ for four different $p_{5} / p_{1}$ values is shown in Fig. 8 . As with Figs. 5 and 6 , the vertical line represents the noise limitation for $c_{3} / c_{2}=0 . \overline{5} 5$. Based on the discussion regarding Fig. $\underline{7}$, the $p_{5} / p_{1}$ ratio should be kept below 0.3 . If these two limitations are adhered to, then the maximum $\beta$ is about 0.25 , which is significantly less than one, as shown in Fig. 8. A similar analysis for the primary driver expansion tail yields that the maximum $\alpha$ is about 0.13 , which is also significantly less than one. The results for this case are presented in Fig. 9. Please note that in both Figs. 8 and $9, \beta$ and $\alpha$ are plotted on logarithmic scales. Therefore, it is a reasonable conclusion to say that within the current operating regions, the primary expansion wave can be neglected as a test-time limitation.

In addition to tube-strength considerations, there is also the requirement of having subatmospheric pressure in the HET facility after each experiment. Impulse facilities such as expansion tubes

Table 2 Selected theoretical test conditions for the HET facility

\begin{tabular}{lccc}
\hline \hline & Air-1 & Air-2 & Air-3 \\
\hline$M_{7}$ & 7.29 & 5.77 & 4.96 \\
$T_{7}, \mathrm{~K}$ & 740 & 1010 & 1080 \\
$p_{7, \mathrm{pitot}}, \mathrm{kPa}$ & 67.1 & 90.4 & 117 \\
$p_{67}, \mathrm{kPa}$ & 0.974 & 2.09 & 3.64 \\
$u_{7}, \mathrm{~m} / \mathrm{s}$ & 3980 & 3670 & 3410 \\
$\rho_{7}, \mathrm{~kg} / \mathrm{m}^{3}$ & 0.005 & 0.007 & 0.012 \\
Test time, $\mu \mathrm{s}$ & 158 & 225 & 293 \\
$c_{3} / c_{2}$ & 0.44 & 0.42 & 0.44 \\
Initial pressures & & & \\
Driver section-, $\mathrm{kPa}$ & 4300 & 4300 & 4300 \\
Driven section, $\mathrm{kPa}$ & 1.5 & 1.2 & 1.2 \\
Expansion section, mtorr & 200 & 500 & 1000 \\
\hline \hline
\end{tabular}

${ }^{\mathrm{a}}$ Based on the burst pressure of a 0.159-cm-thick Al-5052 diaphragm. 


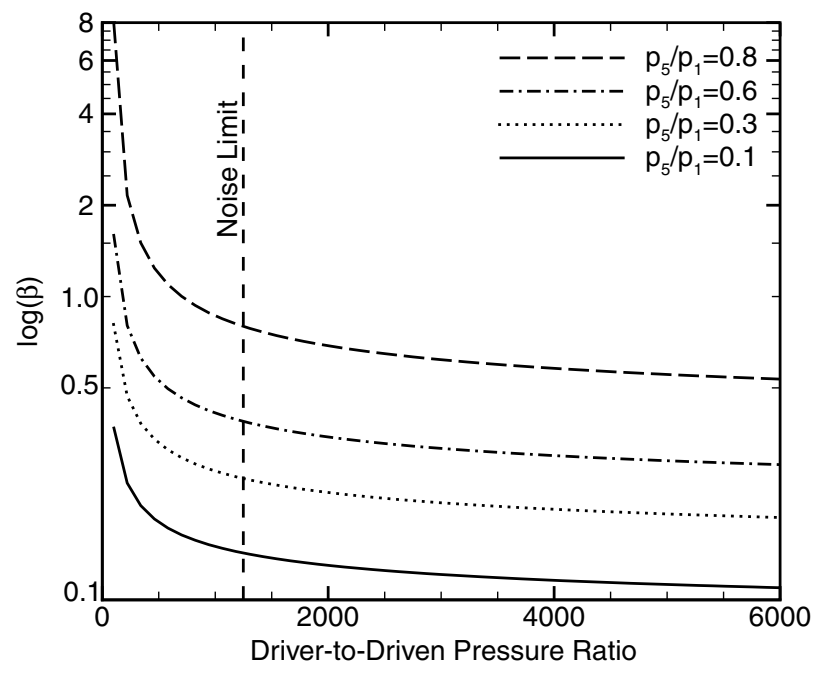

Fig. 8 Ratio $\beta$ as a function of driver-to-driven initial pressure ratio $p_{4} / p_{1}$ for different $p_{5} / p_{1}$ values. The dashed vertical line represents the experimentally identified noise limit.

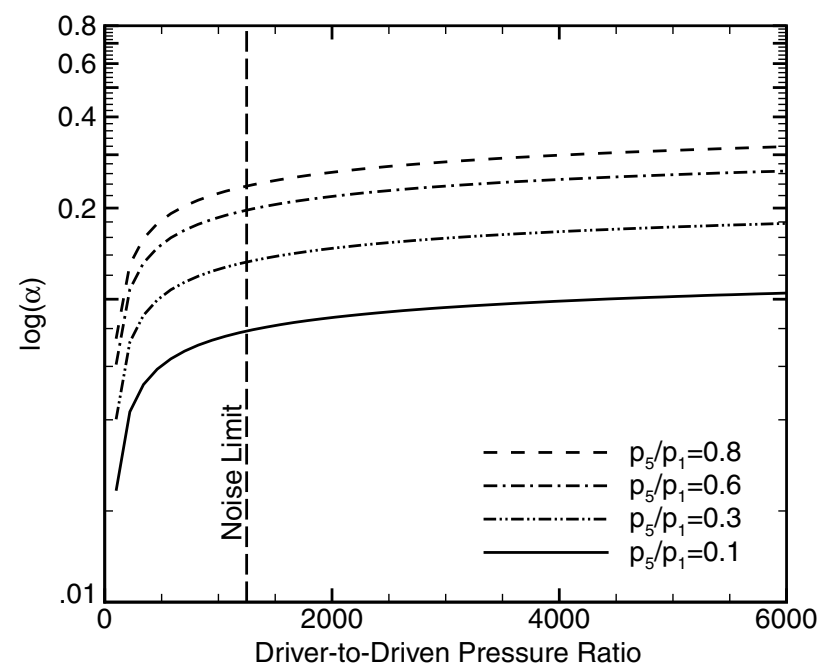

Fig. 9 Ratio $\alpha$ as a function of driver-to-driven initial pressure ratio $p_{4} / p_{1}$ for different $p_{5} / p_{1}$ values; the dashed vertical line represents the experimentally identified noise limit.

typically have an O-ring sliding seal between the tube and the test section. If the postexperiment pressure exceeds atmospheric pressure, this can lead to the rupture of the sliding seal if the tube is not restrained. An order-of-magnitude analysis shows that for the current experiments, the driver fill pressure $p_{4}$ is constrained to be

$$
p_{4}<\frac{p_{\text {atm }} V_{\text {total }}}{V_{\text {driver }}}
$$

where $p_{\text {atm }}$ is atmospheric pressure, $V_{\text {total }}$ is the total volume of the entire facility, and $V_{\text {driver }}$ is the driver volume. It is suggested that a reasonable factor of safety is used in the preceding equation. The current HET design maintains a final facility pressure below $0.85 \mathrm{~atm}$ (factor of safety of 1.17). Although this expression is based on a very simplified analysis, it was found to be sufficient for the design of the dump tank volume, and to prevent sliding-seal rupture for current facility operation.

\section{HET Facility Design and Operation}

The HET facility is constructed of honed $(0.2 \mu \mathrm{m} \mathrm{Ra}) 304 / 304 \mathrm{~L}$ stainless steel with an inner diameter of $152 \mathrm{~mm}$ and a wall thickness of $0.95 \mathrm{~cm}$. Tube lengths were chosen based on the theoretical

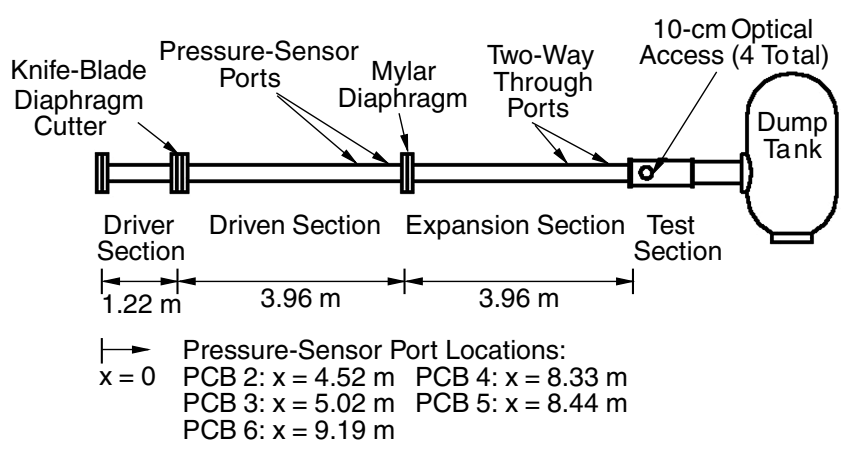

Fig. 10 HET schematic; distances are referenced from the inner wall of the driver.

gasdynamic calculations described in the preceding sections. The length of the driver section is $1.22 \mathrm{~m}$, and the driven and expansion sections are both $3.96 \mathrm{~m}$ long. The tube will withstand initial pressures up to $5500 \mathrm{kPa}$, and the facility is capable of a vacuum of less than 100 mtorr. The facility is mounted on linear bearings for easy access to the diaphragm stations and to allow tube recoil during the experiment. A schematic of the HET is shown in Fig. 10. The test section shown has a sliding seal and four-way $100-\mathrm{mm}$-diam optical access. A sting may be mounted in the test section for pitot probes and models. Ten instrumentation ports are located along the tube, of which four are currently instrumented with piezoelectric pressure transducers for wave time-of-arrival and pressure-profile measurements. A more detailed description of the facility design and experimental setup is reported by Dufrene [18].

\section{A. Primary- and Secondary-Diaphragm Selection}

The primary diaphragm is typically 0.159 -cm-thick $5052-\mathrm{H} 32 \mathrm{Al}$, which has a burst pressure of $4300 \pm 140 \mathrm{kPa}$. Knife blades 11 were found to produce a more repeatable burst pressure in comparison with scored diaphragms or naturally burst diaphragms. End-mill scored diaphragms resulted in variations of $\pm 1000 \mathrm{kPa}$ in the burst pressure. In addition, petal detachment was commonly observed with both naturally burst and scored diaphragms. The knife-blade design was based on the Graduate Aeronautical Laboratories, California Institute of Technology (GALCIT) 17- and 6-in. shock tubes [19]. The knife blades result in a burst-pressure reproducibility of $\pm 1 \overline{40} \mathrm{kPa}$, and the petals always remain intact.

The secondary-diaphragm rupture is an extremely important issue in expansion tube operation, because nonideal rupture interferes with the unsteady expansion wave that produces the secondary acceleration of the test gas. For this reason, the first set of experiments run in the HET facility were used to determine the best material and thickness for the secondary diaphragm. An extensive range of materials was investigated, including aluminum foil, wax paper, and a variety of plastics. The ideal material is one that breaks cleanly and quickly, yet can sustain the initial pressure difference $\left(p_{1}\right.$ to $p_{5}$ ). Aluminum foil and wax paper could not sustain the required pressure difference. The most promising plastics were Mylar and cellophane. Diaphragm opening time is minimized by minimizing diaphragm thickness and maximizing the natural burst pressure [20].

To determine the best material for the secondary diaphragm, several experiments with low driver pressures were conducted. Pressure traces can be seen Fig. 12. The initial pressures for these experiments were 500,10 , and $1 \mathrm{kPa}$ in the driver, driven, and expansion sections, respectively. For the fourth experiment, the driver pressure was doubled. Pressure histories were measured at the third pressure-sensor station in the driven section, which was $0.12 \mathrm{~m}$ from the secondary diaphragm, to investigate the possibility of a reflected shock from that diaphragm. Under ideal operating conditions, no reflected shock exists. Assuming that the secondary diaphragm is replaced by a hard wall, it would take approximately $100 \mu$ s for a reflected shock to reach station 3 in these experiments. As seen in Fig. 12, it actually takes much longer for the reflected wave to appear in the pressure trace, which is consistent with the 


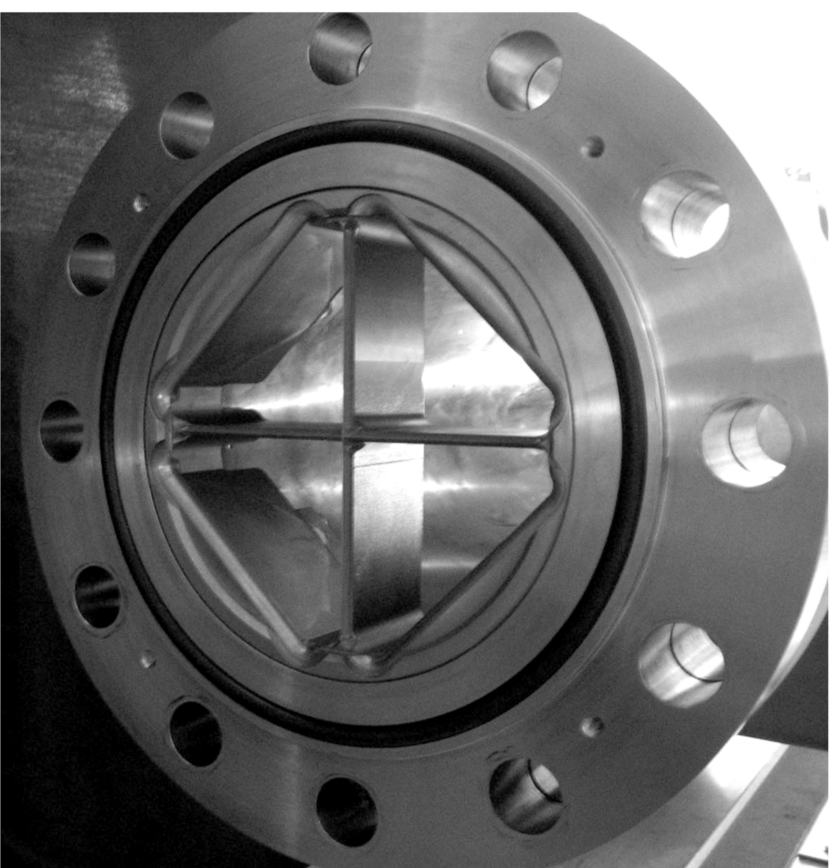

Fig. 11 Knife blades at the primary-diaphragm location.

boundary that actually exists. From these pressure traces, it was determined that the thinner diaphragms and higher driver pressures should produce operating conditions closer to the ideal scenario. Our results compare well with the results from Tohoku University in Japan [21]. They built a transparent test section and looked at the development of the reflected shock front for various thicknesses of Mylar and cellophane diaphragms. They report that a 3- $\mu \mathrm{m}$ Mylar diaphragm resulted in a reflected shock wave that was regarded as a Mach wave, and at that condition, the diaphragm's mechanical influence was practically eliminated. Unfortunately, the two thicknesses of Mylar they used were 25 and $3 \mu \mathrm{m}$, which are an order of magnitude apart. The secondary diaphragm of choice for HET was found to be 12.7- $\mu \mathrm{m}$ Mylar. Heat-treated diaphragms were also investigated. The hope was that through a simple heat treatment, the diaphragms would become more brittle and break more rapidly. No significant improvement was found. In fact, the process occasionally produced small holes in the diaphragm, allowing expansion-section and driven-section gases to mix.

A novel secondary-diaphragm cutter consisting of a thin wire cross (Fig. 13) was mounted downstream of this diaphragm. Before the wire cross, sizable pieces of Mylar diaphragm would be found in the test section after a run. With the addition of the wire cross, fragmentation problems have been resolved without appreciably affecting the test flow.

\section{B. Facility Operation}

The operation of HET begins with selecting a particular run condition and mounting the diaphragms. The 5052-H32 Al diaphragm separates the driver and driven sections, and the $12.7-\mu \mathrm{m}$ Mylar diaphragm separates the driven and expansion sections. Each section has its own manifold, consisting of a gas fill line, a vacuum line, and pressure gauges (MKS Instruments, Inc., Piezo+, KJL205). Because of the low initial pressures in the expansion section, it is evacuated to 500 mtorr or less and then flushed with $10 \mathrm{kPa}$ of helium before evacuation to its operating pressure. The driven and expansion sections must be evacuated simultaneously, ensuring that the pressure difference between the two sections does not exceed $20 \mathrm{kPa}$, to avoid premature rupture of the secondary diaphragm. Once the driven and expansion sections are at the correct pressures, the driver section is evacuated and then pressurized until the primary diaphragm bursts.

A National Instruments modular data acquisition system is used to trigger and collect experimental data. Two dynamic pressure sensors

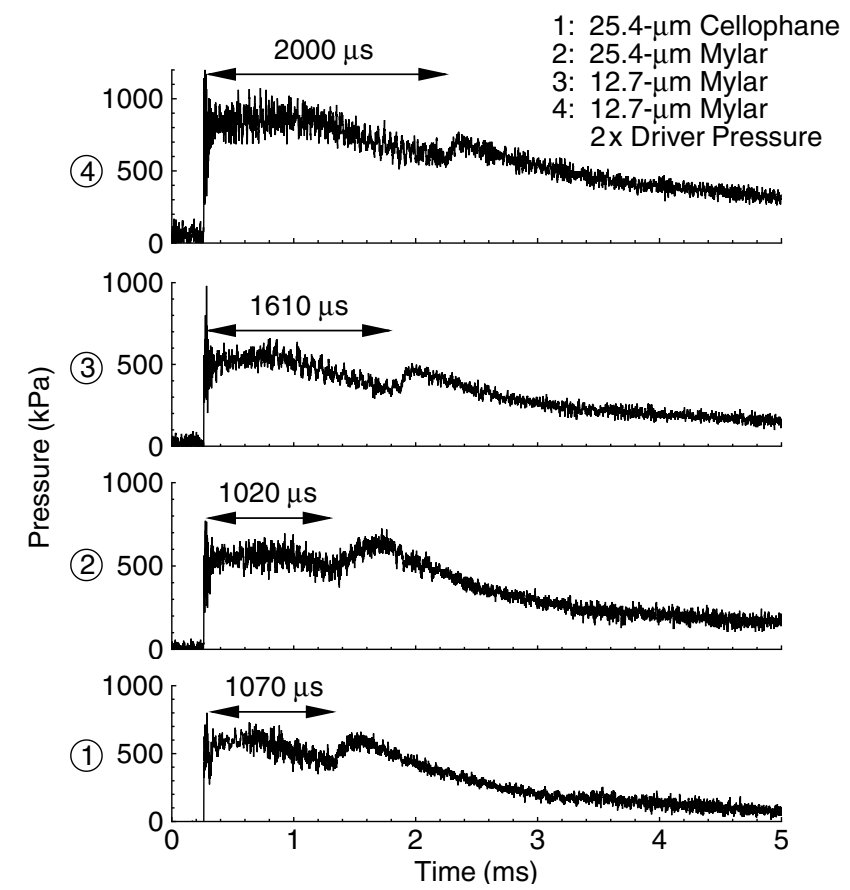

Fig. 12 Pressure traces at pressure-transducer station 3 for various diaphragms; four separate experiments were carried out.

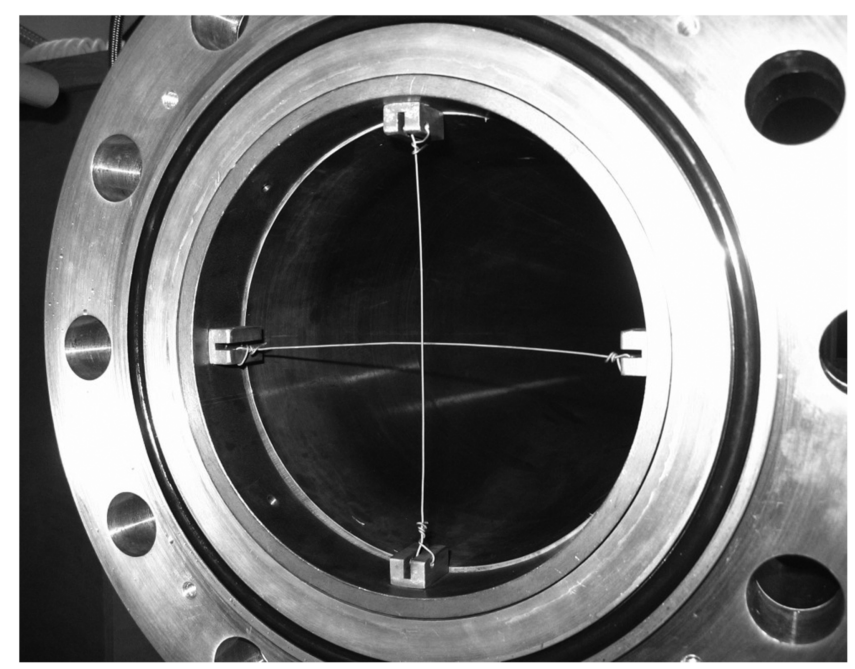

Fig. 13 Wire cross downstream of the secondary diaphragm.

(PCB 113A26) are located in both the driven and expansion sections for pressure and time-of-arrival data. These sensors can also be mounted normal to the flow in the test section for pitot pressure measurements. The sensitivity of these gauges is rated at $\pm 1.45 \mathrm{mV} / \mathrm{kPa}$. A static pressure sensor (Setra 205-2) is located in the driver section to measure the driver pressure before the primary diaphragm ruptures. The accuracy of this gauge is rated at $\pm 27 \mathrm{kPa}$.

A typical pitot trace with air as the test gas and helium as the accelerator gas is shown in Fig. 14. From the pitot pressure, the testgas Mach number $M_{7}$ can be calculated, assuming the pressure recorded is the stagnation pressure behind a normal shock. Figure 14 corresponds to a Mach number of 7.1 at the air-1 run condition. The arrival of the accelerator gas and test gas may be identified as indicated in the figure. The shielded transducers have a minimum response time of $13 \mu \mathrm{s}$, according to Sutcliffe and Morgan [22]. In addition, some test time is lost due to the finite extent of the contact surface. For the example in Fig. 14, the time from the arrival of the contact surface to the beginning of the constant-pressure test gas is $5 \mu \mathrm{s}$. At this time, it is unclear how much of the test time is actually 


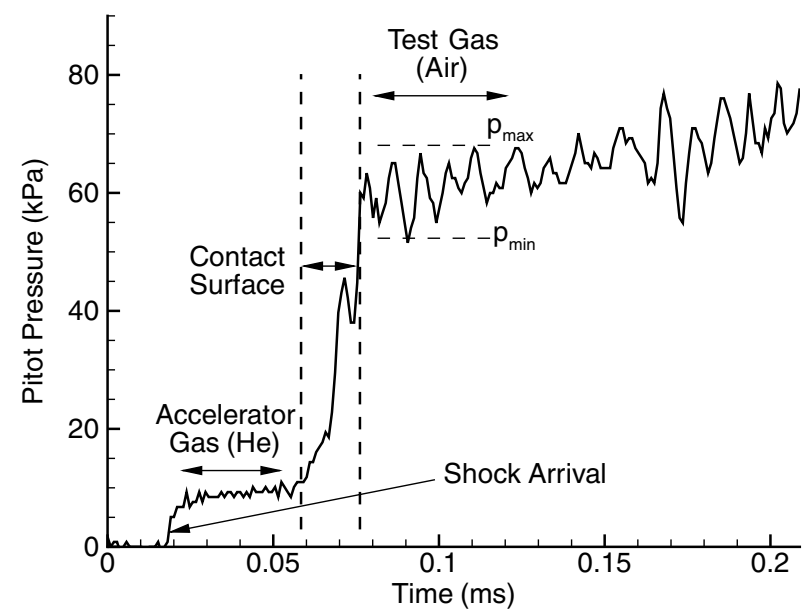

Fig. 14 Typical pitot trace for $M=7.1$ (air-1 run condition).

lost to the contact surface compared with the response time of the shielded transducer. Depending on whether the test time is limited by the arrival of the reflected expansion head or the expansion tail (as discussed in Sec. II), the pressure will either rise or drop after the test time. If the waves arrive around the same time (the optimal condition), the pressure variations tend to cancel each other out and look like typical test-time noise. Future experiments will focus on alternative diagnostics for contact surface arrival measurements.

\section{Experimental Characterization}

Table 3 presents the comparison of experimental data with theoretical predictions for the three run conditions described in Table 2. Pressure transducers located along the tube wall were used for time-of-arrival data to calculate the primary shock velocity. Pitot pressure measurements were made using a dynamic pressure sensor (PCB 113A26) normal to the flow field in the test section. The experimental test time can only be given as an estimate, due to reasons discussed in Sec. III. Shock time-of-arrival data were obtained from pressure transducers mounted along the driven and accelerator sections; however, the postshock pressure in the accelerator section could not be determined, because the signal-tonoise ratio was too poor. Therefore, the experimental Mach number must be calculated using the theoretical prediction of the dynamic pressure within the test section $\left(p_{67}\right)$, along with the experimentally observed $p_{7, \text { pitot }}$ value.

\section{A. Primary Shock Speed $U_{s}$}

It can be seen in Table 3 that the experimental shock speed is consistently measured to be $\overline{2}$ to $5 \%$ below the theoretical prediction. Previous experimental results show that shock speed can vary axially along the tube, rising to a peak above the theoretical value (due to compression waves from the diaphragm opening [23,24]), then decaying below the theoretical value (due to viscous effects $[13,23]$ ). To account for possible axial shock-velocity variation, the shock velocity is measured as close to the secondary-diaphragm station as possible [transducers at $x=5.23$ and $5.28 \mathrm{~m}$ (Fig. 10)]. Results from Table 3 indicate that the pressure transducers are within the viscous attentuation region for all the run conditions considered.

Table 3 HET facility performance: experimental measurements vs theoretical prediction

\begin{tabular}{lcccccc}
\hline \hline & \multicolumn{3}{c}{ Air-1 } & \multicolumn{2}{c}{ Air-2 } & \multicolumn{2}{c}{ Air-3 } \\
\hline & Theory & Exp. & Theory & Exp. & Theory & Exp. \\
Centerline $M_{7}$ & 7.29 & $\mathbf{7 . 1}$ & 5.77 & $\mathbf{5 . 7}$ & 4.96 & $\mathbf{4 . 5}$ \\
$p_{\text {pitot }}, \mathrm{kPa}$ & 67 & $\mathbf{6 4}$ & 90 & $\mathbf{9 0}$ & 117 & $\mathbf{9 2}$ \\
$U_{s}, \mathrm{~m} / \mathrm{s}$ & 2126 & $\mathbf{2 0 6 9}$ & 2187 & $\mathbf{2 1 4 9}$ & 2186 & $\mathbf{2 1 4 0}$ \\
Test time, $\mu \mathrm{s}$ & 158 & $\mathbf{1 0 0}$ & 225 & $\mathbf{1 5 0}$ & 293 & $\mathbf{9 0}$ \\
\hline \hline
\end{tabular}

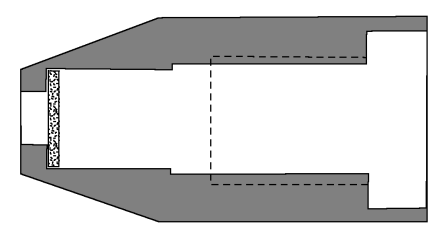

a)

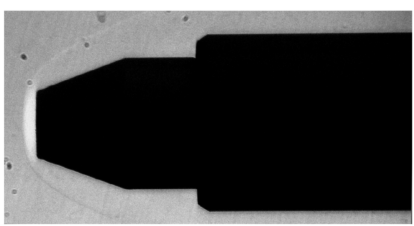

b)

Fig. 15 Schematic of the pressure-transducer mounting for freestream pitot pressure measurements (top) [a porous grill (with nine 1-mm-diam holes) is positioned directly upstream of the transducer diaphragm] and a schlieren image of flow over the pitot probe mounting (bottom).

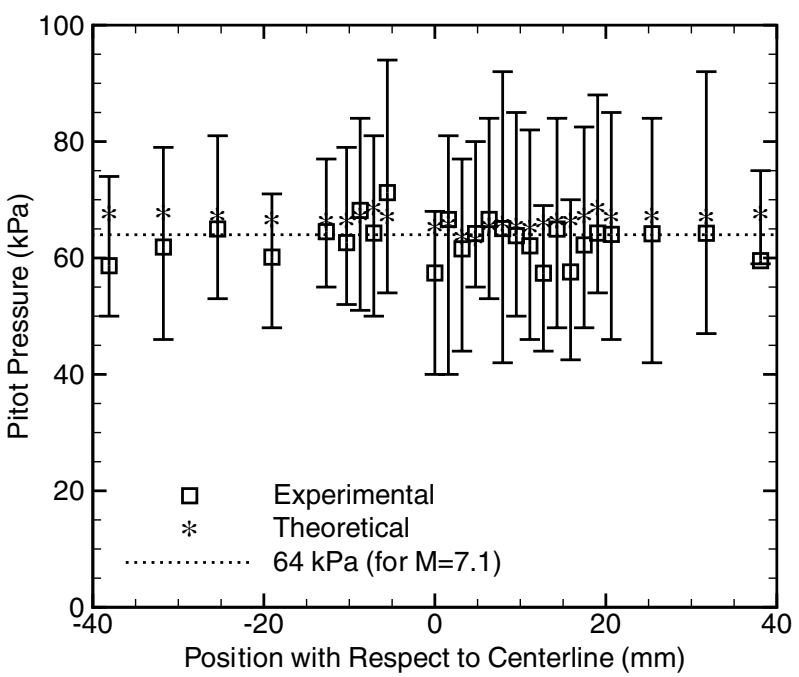

Fig. 16 Pitot survey for the air-1 run condition $31 \mathrm{~mm}$ downstream from the tube exit.

Mechanically induced noise was initially a problem for the wallmounted transducers. To overcome this, the PCB mounting fixtures were redesigned to accommodate two O-rings, which isolate the fixture from the facility wall. This design considerably improved the signal-to-noise ratio.

\section{B. Test-Gas Pitot Profiles and Traces}

Flow disturbances were minimized by following the guidelines suggested by Paull and Stalker [17] and described in Sec. II.B. However, significant pressure oscillations are still present in the pitot pressure traces. The measurement technique and methodology both contribute to the introduction of noise. In the present study, the mounting configuration consists of a protective shell to prevent debris from striking the transducer (Fig. 15). It has been numerically demonstrated that waves form within this shell and create noise that is not within the flow itself [25]. For the nominal $M 7=7.29$ case, pitot pressure surveys were made to determine the size of the core flow. Surveys were conducted at two positions in the test section: 31 and $54 \mathrm{~mm}$ downstream of the tube exit. These pressure surveys are shown in Figs. 16 and 17 . The error bars in the figures are given by the $P_{\max }$ and $P_{\min }$ values that were experimentally measured within the test time (see Fig. 14).

From these profiles, it appears that the axial separation has a minimal effect upon the core flow size over the distance examined. Table $\underline{3}$ shows that the pitot pressures are very close to the theoretical pitot pressures, suggesting that the HET is operating near the 


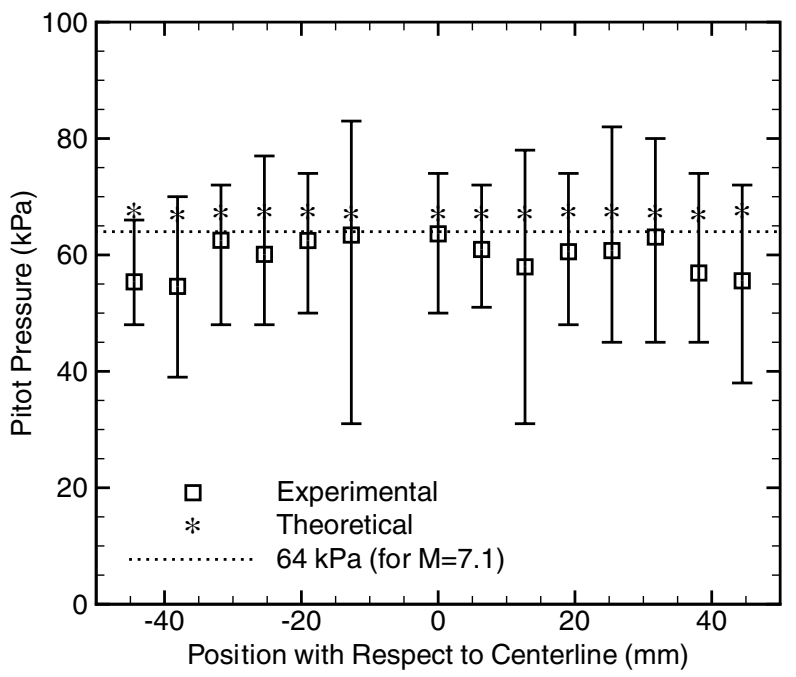

Fig. 17 Pitot survey for air-1 run condition $54 \mathrm{~mm}$ downstream from the tube exit.

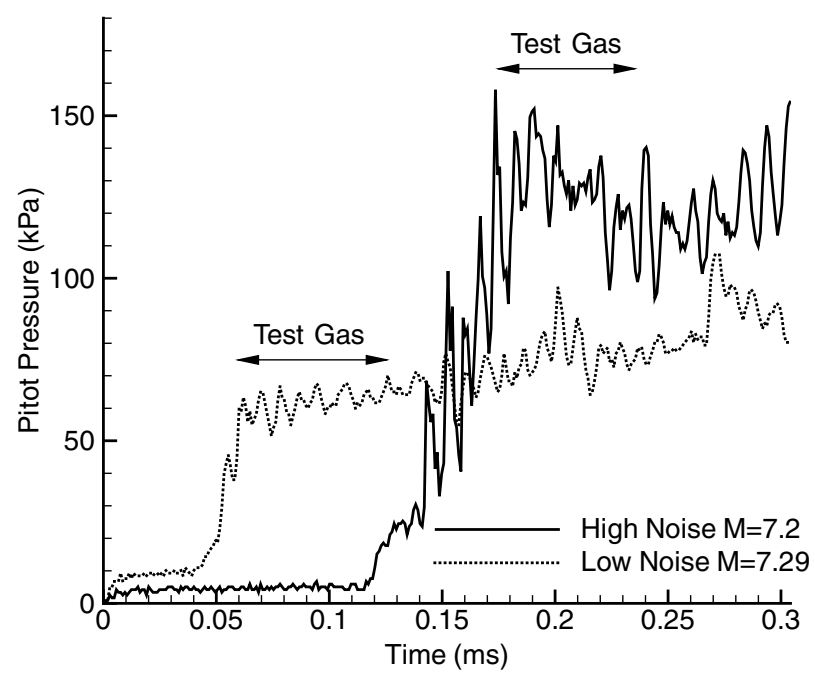

Fig. 18 Pitot histories for nominal Mach 7.29 and 7.20 conditions; $c_{3} / c_{2}$ values are 0.44 and 0.74 , respectively; Mach 7.20 condition had initial pressures of $p_{4}=4300 \mathrm{kPa}, p_{1}=10 \mathrm{kPa}$, and $p_{5}=500 \mathrm{mtorr}$; Mach 7.29 condition is the air-1 condition from Table 2 .

theoretical predictions. A centerline pitot pressure measurement of $64 \mathrm{kPa}$ corresponds to a Mach number of 7.1. Figure 18 shows a comparison between two similar Mach number conditions: a nominal Mach number 7.20 condition and the air-1 run condition with nominal Mach number 7.29. The $c_{3} / c_{2}$ ratios for these two cases are 0.74 and 0.44 , respectively. Visual inspection confirms the postulation that lowering the $c_{3} / c_{2}$ ratio reduces the noise within the test time and that ratios above 0.55 are unacceptable for the current facility.

Despite the variation in driver pressure, HET performance has been very repeatable. In Table 4 , test-gas Mach number repeatability is evaluated from 29 separate shots and 43 pitot samples for the air-1 run condition. Some shots had two pitot probes mounted in the core

Table 4 Test-gas Mach number $M_{7}$ repeatability for the air-1 run condition

\begin{tabular}{ll}
\hline \hline Average Mach number & 7.05 \\
Standard deviation & 0.21 \\
Run-to-run percent difference & 3.04 \\
Theoretical prediction variation, \% & 3.61 \\
Standard deviation of theoretical variation & 2.60 \\
\hline \hline
\end{tabular}

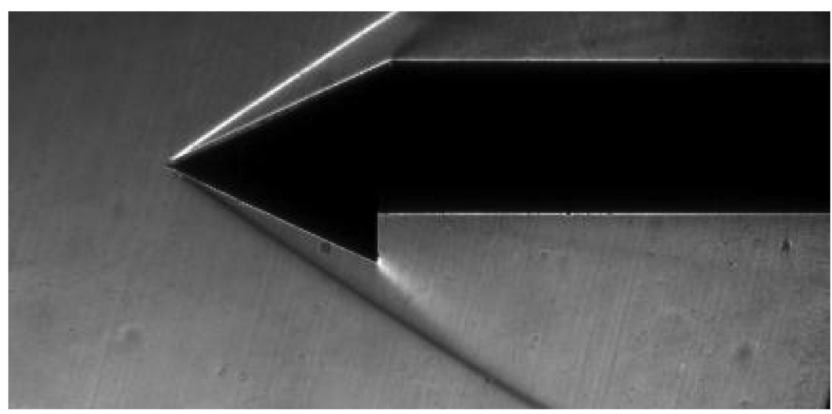

Fig. 19 Schlieren image of a 25-deg wedge at the air-1 run condition.

flow for the test, explaining the discrepancy between number of shots and number of pitot samples evaluated. Only samples within the core flow defined in Sec. IV.B were used. The ideal Mach number varies slightly from run to run, based on the initial driver pressure. For this example, the run-to-run percent difference is roughly $3 \%$, and the percent difference from ideal theoretical predictions is only $3.61 \%$.

\section{Flow over Wedge Geometries}

Schlieren images were taken of the flow over wedge geometries to infer the Mach number from the shock angle. The theoretical shock angle is given by the well-known $\theta-\beta-M$ relationship.

Figure 19 shows an image taken at the air- 1 run condition. The experimental Mach number was 7.1 from the pitot data, and the measured shock angle was $33.65 \mathrm{deg}$ from the schlieren image. A shock angle of $33.12 \mathrm{deg}$ corresponds to a nominal perfect-gas Mach number of 7.29, and a shock angle of $33.23 \mathrm{deg}$ corresponds to the experimentally measured Mach number of 7.1. The measured shock angle of $33.65 \mathrm{deg}$ corresponds to a flow Mach number of 6.57. There was a $\pm 0.8 \%$ error in measuring the shock angle.

\section{Conclusions}

Expansion tubes have the potential to offer a wide range of highstagnation-enthalpy test flow conditions relevant to hypervelocity propulsion systems. This range of test conditions may be limited in practice by insufficient test time and core flow size or substantial flow disturbances. A generalized design strategy for small-scale expansion tubes is presented. The procedure was used to design an expansion tube facility capable of producing a range of reasonablequality test flows with Mach numbers over the range of 3.0 to 7.1.

Gasdynamic calculations of expansion tube operation show that the maximum test time for high-enthalpy operating conditions is obtained when the tail of the expansion wave and the leading characteristic of the reflected expansion coincide. We find that the interaction of the two expansion waves significantly delays the arrival of the leading characteristic, and accounting for this effect may increase the theoretical test time by as much as $35 \%$.

Lateral acoustic waves generated in the driver section were found to be a significant source of these disturbances. The penetration of acoustic waves into the test gas can be minimized by minimizing the ratio of the expanded driver gas to the driven-gas sound speed. We find that the sound speed ratio $c_{3} / c_{2}$ should be less than 0.55 to reduce the measured pitot pressure peak-to-peak fluctuations to less than $50 \%$ in our facility. The quality of the core flow is expected to be somewhat better than this value, because the shroud-and-cavity mounting used to protect the transducers in this measurement is expected to contribute to the observed pressure oscillations.

Within these limits, the test flow Mach number and maximum test time can be selected by varying the initial pressures in each of the three sections of the facility. The test flow Mach number $M_{7}$ is a relatively strong function of the accelerator-to-driven-section pressure ratio $p_{5} / p_{1}$, particularly for higher Mach numbers (greater than about four), which correspond to $p_{5} / p_{1}$ values less than about $0.2 . M_{7}$ is only weakly dependent on the driver-to-driven-gas pressure ratio $p_{4} / p_{1}$ for the conditions considered in this study. The test time is also relatively independent of the driver-to-driven-gas 
pressure ratio for $p_{4} / p_{1}$ greater than about 1000 . Because values of $p_{4} / p_{1}$ less than 1000 correspond to sound speed ratios with largeamplitude flow disturbances, the test time is determined predominately by the accelerator-to-driven-section pressure ratio $p_{5} / p_{1}$. Decreasing the $p_{5} / p_{1}$ ratio increases the test time; however, the test flow Mach number is also decreased. Thus, the optimal initial pressures can be selected based on specific experimental requirements.

The preceding procedure was used to design the HET. The HET consists of three sections: a 1.22-m driver, a 3.96-m driven tube, and a 3.96-m expansion tube, all of honed stainless steel tubing with an inner diameter of $152 \mathrm{~mm}$. The exit of the accelerator tube is connected to a test section with four-way 100-mm-diam optical ports via a sliding seal. The HET facility is fully operational and capable of being run multiple times per day.

A knife-blade cross is used to burst the primary diaphragm. The knife blades have a much more reproducible burst pressure of $\pm 140 \mathrm{kPa}$ compared with scored diaphragms, which varied by $\pm 1000 \mathrm{kPa}$. The knife blades also achieve higher burst pressures than the scored diaphragms, without the loss of diaphragm petals.

The influence of the secondary diaphragm is an important issue for expansion tubes. Pressure data were used to investigate the effect of several secondary-diaphragm materials and thicknesses on the quality of the test flow, and 12.7- $\mu \mathrm{m}$ Mylar was selected for all of the current run conditions. Additionally, a novel crossed-wire arrangement was found to result in repeatable test conditions and reduced diaphragm fragmentation without adding appreciable disturbances to the test flow.

A range of test conditions (Mach 3.0 to 7.1) were verified through pitot pressure measurements and shock-angle measurements over simple geometries. Experimental conditions are compared with gasdynamic calculations and found to be in good agreement. Pitot pressure surveys at two downstream locations (31 and $54 \mathrm{~mm}$ downstream of the tube exit) were used to measure the core flow size. The flow is uniform over approximately $60 \mathrm{~mm}$ for the Mach 7.1 test condition.

\section{Acknowledgments}

This research was funded in part through a Multi-University Research Initiative (MURI) granted by the U.S. Air Force Office of Scientific Research (AFOSR), with John Schmisseur as Technical Monitor. Special thanks to Matthew D. Parker for test-section design and construction and data acquisition system installation, to Matthew McGilvray for the design of the transducer mounts, and to Richard Morgan for helpful discussions.

\section{References}

[1] Hornung, H. G., "Experimental Hypervelocity Flow Simulation, Needs, Achievements, and Limitations," Proceedings of the 1st Pacific International Conference on Aerospace Science and Technology, National Cheng-Kung Univ., Tainan, Taiwan, 1994, pp. 1-10.

[2] Lukasiewicz, J., Experimental Methods of Hypersonics, Marcel Dekker, New York, 1973.

[3] Lu, F. K., and Marren, D. E. (eds.), Advanced Hypersonic Test Facilities, Vol. 198, Progress in Astronautics and Aeronautics Series, AIAA, Reston, VA, 2002.

[4] Hornung, H. G., "Performance Data of the New Free-Piston Shock Tunnel at GALCIT," AIAA Paper 92-3943, 1992.

[5] Albrechcinski, T., Boyer, D., Chadwick, K., and Lordi, J., "Calspan's Upgraded 96-Inch Hypersonic Shock Tunnel: Its Development and
Application in Performance of Research and Testing at Higher Enthalpies," AIAA Paper 95-0236, 1995.

[6] Hanneman, K., and Beck, W., "Aerothermodynamics Research in the DLR High Enthalpy Shock Tunnel HEG," Advanced Hypersonic Test Facilities, edited by F. K. Lu and D. E. Marren, Vol. 198, Progress in Astronautics and Aeronautics Series, AIAA, Reston, VA, 2002, pp. 205-238.

[7] Itoh, K., Ueda, S., Tanno, H., Komuro, T., and Sato, K., "Hypersonic Aerothermodynamic and Scramjet Research Using High Enthalpy Shock Tunnel," Shock Waves, Vol. 12, No. 2, 2002, pp. 93-98.

[8] Erdos, J. I., Bakos, R. J., Castrogiovanni, A., and Rogers, R. C., "Dual Mode Shock Expansion/Reflected Shock Tunnel," AIAA Paper 970560, 1997.

[9] Sasoh, A., Ohnishi, Y., Ramjaun, D., Takayama, K., Otsu, H., and Abe, T., "Effective Test Time Evaluation in High-Enthalpy Expansion Tube," AIAA Journal, Vol. 39, No. 11, 2001, pp. 2141-2147.

[10] Neely, A. J., and Morgan, R. G., "The Superorbital Expansion Tube Concept, Experiment and Analysis," The Aeronautical Journal, Vol. 98, No. 973, 1994, pp. 97-105.

[11] Heltsley, W. N., Snyder, J. A., Houle, A. J., Davidson, D. F., Mangal, M. G., and Hanson, R. K., "Design and Characterization of the Stanford 6 Inch Expansion Tube," AIAA Paper 06-4443, 2006.

[12] Trimpi, R. L., "Preliminary Theoretical Study of the Expansion Tube, New Device for Producing High Enthalpy Short-Duration Hypersonic Gas Flows," NASA TR R-133, 1962.

[13] Shinn, J. L., and Miller, C. G., "Experimental Perfect Gas Study of Expansion-Tube Flow Characteristics," NASA TP 1317, 1978.

[14] Wintenberger, E., Austin, J. M., Cooper, M., Jackson, S., and Shepherd, J. E., "An Analytical Model for the Impulse of a Single-Cycle Pulse Detonation Engine," Journal of Propulsion and Power, Vol. 19, No. 1, 2003, pp. 22-38.

[15] Spurk, J. H., "Design, Operation and Preliminary Results of the BRL Expansion Tube," Fourth Hypervelocity Techniques Symposium, Ballistic Research Labs., Aberdeen Proving Ground, MD, 1965, pp. 114-144.

[16] Norfleet, G. D., Lacey, J. J., and Whitfield, J. D., "Results of an Experimental Investigation of the Performance of an Expansion Tube," Fourth Hypervelocity Techniques Symposium, Ballistic Research Labs., Aberdeen Proving Ground, MD, 1965, pp. 49-110.

[17] Paull, A., and Stalker, R. J., "Test Flow Disturbances in an Expansion Tube," Journal of Fluid Mechanics, Vol. 245, Dec. 1992, pp. 493-521.

[18] Dufrene, A., "Design and Characterization of the Hypervelocity Expansion Tube," M.S. Thesis, Univ. of Illinois at Urbana-Champaign, Urbana, IL, 2006.

[19] Roshko, A., and Baganoff, D., "A Novel Device for Bursting ShockTube Diaphragms," Physics of Fluids, Vol. 4, No. 11, 1961, pp. 14451446.

[20] Rothkopf, E. M., and Low, W., "Diaphragm Opening Process in Shock Tubes," Physics of Fluids, Vol. 17, No. 6, 1974, pp. 1169-1173.

[21] Furukawa, T., Aochi, T., and Sasoh, A., "Expansion Tube Operation with Thin Secondary Diaphragm," AIAA Paper 06-1097, 2006.

[22] Sutcliffe, M. A., and Morgan, R. G., "The Measurement of Pitot Pressure in High Enthalpy Expansion Tubes," Measurement Science and Technology, Vol. 12, No. 3, 2001, pp. 327-334.

[23] Ikui, T., Matsuo, K., and Nagai, M., "Investigations of the Aerodynamic Characteristics of the Shock Tubes, Part 2: On the Formation of Shock Waves)," Bulletin of JSME, Vol. 12, No. 52, 1969, pp. 783-792.

[24] White, D. R., "Influence of Diaphragm Opening Time on Shock-Tube Flows," Journal of Fluid Mechanics, Vol. 4, No. 6, 1958, pp. 585-599.

[25] Buttsworth, D. R., and Jacobs, P., "Measurement of Fluctuations in a Mach 4 Shock Tunnel Nozzle Flow," Proceedings of the 7th Australasian Heat and Mass Transfer Conference, Chalkface Press, Perth, Western Australia, 2000, pp. 53-59.
R. Bowersox Associate Editor 